\title{
Dense properties of the space of the circle diffeomorphisms with a Liouville rotation number
}

\author{
Shigenori Matsumoto
}

\begin{abstract}
Given any Liouville number $\alpha$, it is shown that various subspaces are $C^{\infty}$-dense in the space of the orientation preserving $C^{\infty}$ diffeomorphisms of the circle with rotation number $\alpha$.
\end{abstract}

\section{Introduction}

Denote by $F$ the group of the orientation preserving $C^{\infty}$ diffeomorphisms of the cirlce. For $\alpha \in \mathbb{R} / \mathbb{Z}$, denote by $F_{\alpha}$ the subspace of $F$ consisting of all the diffeomorphisms with rotation number $\alpha$. If $\alpha$ is irrational, a famous theorem of A. Denjoy [D] states that any element $f$ of $F_{\alpha}$ is conjugate to the rotation by $\alpha$, denoted $R_{\alpha}$, by an orientation preserving homeomorphism. Precisely, there is a unique orientation preserving homeomorphism $H_{f}$ of $S^{1}$ such that

$$
f=H_{f} R_{\alpha} H_{f}^{-1} \text { and } H_{f}(0)=0 .
$$

Now denote by $O_{\alpha}$ the subspace of $F_{\alpha}$ of all the diffeomorphisms for which $H_{f}$ are $C^{\infty}$-diffeomorphisms. In [Y1], J.-C. Yoccoz showed that $O_{\alpha}=F_{\alpha}$ if $\alpha$ is a non-Liouville number. He also obtained ([Y2]) the following $C^{\infty}$-density result even for a Liouville number $\alpha$.

Theorem 1.1. For any irrational number $\alpha$, the space $O_{\alpha}$ is $C^{\infty}$-dense in $F_{\alpha}$.

Henceforth in this paper we assume $\alpha$ is a Liouville number. Our purpose is to show that subspaces of $F_{\alpha}$ which are defined by intermediate regularities of $H_{f}$ are also $C^{\infty}$-dense in $F_{\alpha}$. But first of all let us state fundamental facts about them.

Remark 1.2. ([H] , Chapter IV, Sect. 6)

If $H_{f}$ is a bi-Lipschitz homeomorphism, then it is a $C^{1}$-diffeomorphism.

The unique $f$-invariant probability measure $\mu_{f}$ is given by

$$
\mu_{f}=\left(H_{f}\right)_{*} m,
$$

where $m$ is the Lebesgue measure on $S^{1}$. The uniqueness of $\mu_{f}$, together with the ergodicity of $m$ w. r. t. $f\left([\mathbf{H}]\right.$, p.86), implies that either $\mu_{f}$ is equivalent to $m$ or singular to $m$. From this follows easily the following remark.

1991 Mathematics Subject Classification. Primary 37E10, secondary 37E45.

Key words and phrases. circle diffeomorphism, rotation number, Liouville number, conjugacy, invariant measure, fast approximation by conjugation.

The author is partially supported by Grant-in-Aid for Scientific Research (C) No. 20540096. 
Remark 1.3. (i) If $\mu_{f}$ is equivalent to $m$, then the homeomorphism $H_{f}$ maps any Lebesgue null set to a null set.

(ii) If $\mu_{f}$ is singular to $m$, then $H_{f}$ maps some Lebesgue null set to a conull set.

In case (i), the conjugacy $H_{f}$ is called absolutely continuous, and in case (ii) singular.

Besides this distinction, there are Hölder conditions. Recall that a homeomorphism $H: S^{1} \rightarrow S^{1}$ is called $\beta$-Hölder $(\beta \in(0,1])$ if there is a constant $C>0$ such that for any $x, y \in S^{1}$,

$$
|H(x)-H(y)| \leq C|x-y|^{\beta} .
$$

Let us define subspaces of $F_{\alpha}$ according to the regularity of $H_{f}$ w. r. t. these two measures. But we shall omit the subspace corresponding to the classes between Lipschitz and $C^{1}$, because it is empty by Remark 1.2. We shall list the name of the subspaces of $F_{\alpha}$ and the corresponding properties of $H_{f}$.

Definition 1.4. Let $\beta \in(0,1)$ and $k \in \mathbb{N}$.

- $G_{0, \text { sing }}: H_{f}$ is singular and is not $d$-Hölder for any $d \in(0,1)$.

- $G_{0, \text { ac }}: H_{f}$ is absolutely continuous and is not $d$-Hölder for any $d \in(0,1)$.

- $G_{\beta}: H_{f}$ is bi $\beta$-Hölder but is not $d$-Hölder for any $d \in(\beta, 1)$.

- $G_{1, \text { sing }}: H_{f}$ is singular and is bi $d$-Hölder for any $d \in(0,1)$.

- $G_{1, \text { ac }}: H_{f}$ is absolutely continuous and is $d$-Hölder for any $d \in(0,1)$, but is not bi-Lipschitz.

- $G_{k}: H_{f}$ is a $C^{k}$ diffeomorphism, but is not a $C^{k+1}$ diffeomorphism.

The subspaces in Definition 1.4 are mutually disjoint. Our main theorem is the following.

Theorem 1. For any Liouville number $\alpha$, the subspaces defined in Definition 1.4 are $C^{\infty}$-dense in $F_{\alpha}$.

We are not going to prove the $C^{\infty}$-density of $G_{0, \text { sing }}$, which is already done in M. Moreover it is shown there that the subspace of $f \in F_{\alpha}$ such that the Hausdorff dimension of $\mu_{f}$ is 0 contains a countable union of $C^{0}$-open and $C^{\infty}$-dense subsets of $F_{\alpha}$. On the other hand it is known ( $\mathbf{Y} \mathbf{3},[\mathbf{S}]$ ) that the nullity of the Hausdorff dimension of $\mu_{f}$ implies that $\mu_{f}$ is singular and that $H_{f}^{-1}$ is not $d$-Hölder for any $d \in(0,1)$. Therefore we have:

Remark 1.5. ( $\mathbf{M}]$ ) For any Liouville number $\alpha$, the space $G_{0, \text { sing }}$ contains a countable intersection of $C^{0}$-open and $C^{\infty}$-dense subspaces, and in particular is residual in the $C^{r}$-topology $(r=0,1, \cdots, \infty)$.

Remark 1.6. The subspace $G_{\beta}$ can be divided into $G_{\beta, \text { sing }}$ and $G_{\beta, \text { ac }}$, and the both subspaces seem to be $C^{\infty}$-dense. But we do not pursue this problem.

Theorem 1 is proved by the method of fast approximation by conjugacy with estimate, developed in FS. Moreover all the cases can be treated by a more or less unified fashion. Sect. 2 is devoted to the exposition of this method. Each space in Definition 1.4 is dealt with separately in further sections.

The author does not claim much originality of the arguments here. For example, V. Sadovskaya $(\underline{\mathbf{S}}])$ showed that for any Liouville number $\alpha$ and $d \in[0,1]$ there 
exists a diffeomorphism $f \in F_{\alpha}$ for which the Hausdorff dimension of $\mu_{f}$ is $d$. A modification of her argument yields a proof for the density of $G_{\beta}$. The space $G_{k}$ is dealt with by elaborations of arguments found in $[\mathbf{K H}, 12.6$.

But Theorem 1 seems to be worth recording, while the author cannot find it in the literature. This determines him to write down this paper.

\section{Fast approximation method}

2.1. Overview. We assume throughout that $0<\alpha<1$ is a Liouville number, i. e. for any $N \in \mathbb{N}$ and $\epsilon>0$, there is $p / q(p, q \in \mathbb{N},(p, q)=1)$ such that

$$
|\alpha-p / q|<\epsilon q^{-N} \text {. }
$$

Let $G$ be one of the subsets of diffeomorphisms of $F_{\alpha}$ defined in Definition 1.4 To show that $G$ is $C^{\infty}$-dense in $F_{\alpha}$, it suffices to establish the following proposition. We denote by $d_{r}$ the $C^{r}$-metric on $F$, to be defined precisely later.

Proposition 2.1. For any $r \in \mathbb{N}$, there exists $f \in G$ such that $d_{r}\left(f, R_{\alpha}\right)<2^{-r}$.

In fact Proposition 2.1 implies that $R_{\alpha}$ is contained in the $C^{r}$-closure of $G$ for any $r$, and therefore in the $C^{\infty}$-closure of $G$, denoted $\mathrm{Cl}_{\infty}(G)$. Since $\mathrm{Cl}_{\infty}(G)$ is invariant by the conjugation by any $h \in F$, it follows that

$$
O_{\alpha} \subset \mathrm{Cl}_{\infty}(G) \text {. }
$$

Then by virtue of Theorem 1.1, we have

$$
F_{\alpha}=\mathrm{Cl}_{\infty}(G) .
$$

To prove Proposition 2.1 for $G$, we will actually show the next proposition.

Proposition 2.2. For any $r \in \mathbb{N}$, there are sequences $\alpha_{n}=p_{n} / q_{n}$ converging to $\alpha$ and $h_{n} \in F(n \in \mathbb{N})$ such that the following (i), (ii) and (iii) hold. Define $H_{0}=\mathrm{Id}$, $f_{0}=R_{\alpha_{1}}$, and for any $n \in \mathbb{N}$

$$
H_{n}=h_{1} \cdots h_{n} \text { and } f_{n}=H_{n} R_{\alpha_{n+1}} H_{n}^{-1} .
$$

(i) $R_{\alpha_{n}}$ commutes with $h_{n}$.

(ii) $H_{n}^{-1}$ converges uniformly to a homemorphism $H^{-1}$ and its inverse $H=H_{f}$ satisfies the correspoing properties for $G$ listed in Definition 1.4

$$
\left|\alpha-\alpha_{1}\right|<2^{-r-1}, \text { and } d_{n+r}\left(f_{n-1}, f_{n}\right)<2^{-n-r-1}, \quad \forall n \geq 1 .
$$

Notice that (iii) implies that the limit $f$ of $f_{n}$ is a $C^{\infty}$ diffeomorphism. It also satisfies $d_{r}\left(f, R_{\alpha}\right)<2^{-r}$. On the other hand the limits $H^{-1}$ and $f$ satisfy $H^{-1} f=R_{\alpha} H^{-1}$. That is,

$$
f=H R_{\alpha} H^{-1} .
$$

This shows that Proposition 2.1 is satisfied by $f \in F_{\alpha}$.

Condition (i) is useful to establish (iii), since then

$$
\begin{aligned}
f_{n-1}-f_{n} & =H_{n} R_{\alpha_{n}} H_{n}^{-1}-H_{n} R_{\alpha_{n+1}} H_{n}^{-1}, \\
f_{n-1}^{-1}-f_{n}^{-1} & =H_{n} R_{-\alpha_{n}} H_{n}^{-1}-H_{n} R_{-\alpha_{n+1}} H_{n}^{-1},
\end{aligned}
$$

and these can be estimated using Lemma 2.4 below. 
2.2. Estimates. Next we shall summerize inequalities needed to establish Proposition 2.2. All we need are polynomial type estimates whose degree and coefficients can be arbitrarily large. The inequalities below are sometimes far from being optimal. by

For a $C^{\infty}$ function $\varphi$ on $S^{1}$, we define as usual the $C^{r}$ norm $\|\varphi\|_{r}(0 \leq r<\infty)$

$$
\|\varphi\|_{r}=\max _{0 \leq i \leq r} \sup _{x \in S^{1}}\left|\varphi^{(i)}(x)\right|
$$

For $f, g \in F$, define

$$
\begin{aligned}
\||f|\|_{r} & =\max \left\{\|f-\mathrm{id}\|_{r},\left\|f^{-1}-\mathrm{id}\right\|_{r}, 1\right\}, \\
d_{r}(f, g) & =\max \left\{\|f-g\|_{r},\left\|f^{-1}-g^{-1}\right\|_{r}\right\} .
\end{aligned}
$$

The term $\||f|\|_{r}$ is used to show that $f$ is not so big in the $C^{r}$-topology. On the other hand $d_{r}(f, g)$ is useful for showing $f$ and $g$ are near in the $C^{r}$-topology. The following inequality follows easily from the Faà di Bruno formula $(\mathbf{H}]$, p.42 or $[\mathbf{S}]$ ). We have included 1 in the definition of $\||f|\|_{r}$ in order to have

$$
\||f|\|_{r}^{i} \leq\||f|\|_{r}^{r} \text { if } i \leq r .
$$

Below we denote by $C(r)$ an arbitrary constant which depends only on $r$.

Lemma 2.3. For $f, g \in F$ we have

$$
\begin{aligned}
\|f g-g\|_{r} & \leq C(r)\|f-\mathrm{Id}\|_{r}\||g|\|_{r}^{r}, \\
\||f g|\|_{r} & \leq C(r)\||f|\|_{r}^{r}\||g|\|_{r}^{r} .
\end{aligned}
$$

The next lemma can be found as Lemma 5.6 of $[\mathbf{F S}]$ or as Lemma 3.2 of $[\mathbf{S}]$.

Lemma 2.4. For $H \in F$ and $\alpha, \beta \in \mathbb{R} / \mathbb{Z}$,

$$
d_{r}\left(H R_{\alpha} H^{-1}, H R_{\beta} H^{-1}\right) \leq C(r)\||H|\|_{r+1}^{r+1}|\alpha-\beta| .
$$

For $q \in \mathbb{N}$, denote by $\pi_{q}: S^{1} \rightarrow S^{1}$ the cyclic $q$-fold covering map.

Lemma 2.5. Let $h$ be a lift of $\hat{h} \in F$ by $\pi_{q}$ and assume $\operatorname{Fix}(h) \neq \emptyset$. Then we have for any $r \geq 0$

$$
\begin{aligned}
\|h-\mathrm{Id}\|_{r} & =\|\hat{h}-\mathrm{Id}\|_{r} q^{r-1}, \\
\||h|\|_{r} & \leq\|\hat{h} \mid\|_{r} q^{r-1} .
\end{aligned}
$$

Proof. Just notice that a lift $\tilde{h}$ of $h$ to $\mathbb{R}$ is the conjugate of a lift $\tilde{\hat{h}}$ of $\hat{h}$ by a homothety by $q$, i. e. $\tilde{h}(x)=q^{-1} \tilde{\hat{h}}(q x)$.

Let us explain more concretely the way to construct the homeomorphisms $h_{n}$ and the rationals $\alpha_{n}=p_{n} / q_{n}$. Essentially there are two methods. 
2.3. Method I. Here we shall expose a method whose idea is easy to understand and applicable to $G_{1 \text {,sing }}$ and $G_{1, \text { ac }}$. First of all we construct beforehand a sequence of diffeomorphisms $\hat{h}_{n} \in F$ with nonempty fixed point set; $\operatorname{Fix}\left(\hat{h}_{n}\right) \neq \emptyset$. Next we choose a sequence of rationals $\alpha_{n}=p_{n} / q_{n}$ in a way to be explained below, and set $h_{n}$ to be the lift of $\hat{h}_{n}$ by the cyclic $q_{n}$-fold covering map such that $\operatorname{Fix}\left(h_{n}\right) \neq \emptyset$. Then condition (i) of Proposition 2.2 is automatically satisfied.

We always choose the rationals $\alpha_{n}$ so as to satisfy

$$
\left|\alpha_{n+1}-\alpha\right|<\left|\alpha_{n}-\alpha\right|, \quad \forall n \in \mathbb{N} .
$$

Therfore we have

$$
\left|\alpha_{n}-\alpha_{n+1}\right| \leq 2\left|\alpha-\alpha_{n}\right|
$$

We shall discuss how to define $\alpha_{n}$ to garantee condition (iii). It is by induction on $n$. Here we denote any constant which depends on $r, \hat{h}_{i}(1 \leq i \leq n)$ and $\alpha_{1}, \cdots, \alpha_{n-1}$ by $C(n, r)$. Thus $C(n, r)$ is any constant depending only on the innitial data about $h_{i}$ and the previous step of the induction. We also denote any positive integer which depends on $n$ and $r$ by $N(n, r)$.

By Lemma 2.5. we have for any $1 \leq i<n$,

and

$$
\left\|\left|h_{i}\right|\right\|_{n+r+1} \leq\left\|\left|\hat{h}_{i}\right|\right\|_{n+r+1} q_{i}^{n+r}=C(n, r)
$$

$$
\left\|\left|h_{n}\right|\right\|_{n+r+1} \leq\left\|\left|\hat{h}_{n}\right|\right\|_{n+r+1} q_{n}^{n+r}=C(n, r) q_{n}^{N(n, r)} .
$$

Of course the two $C(n, r)$ 's in (2.2) and (2.3) are different. Now we obtain inductively using Lemma 2.3 that

$$
\left\|\left|H_{n}\right|\right\|_{n+r+1} \leq C(n, r) q_{n}^{N(n, r)} .
$$

The terms $C(n, r)$ and $N(n, r)$ in (2.4) are computed from (2.2) and (2.3) by applying Lemma 2.3 successively. Then by Lemma 2.4 and (2.4),

$$
\begin{aligned}
d_{n+r}\left(f_{n-1}, f_{n}\right) & =d_{n+r}\left(H_{n} R_{\alpha_{n}} H_{n}^{-1}, H_{n} R_{\alpha_{n+1}} H_{n}^{-1}\right) \\
& \leq C(n, r) q_{n}^{N(n, r)}\left|\alpha_{n}-\alpha_{n+1}\right| \\
& \leq C(n, r) q_{n}^{N(n, r)}\left|\alpha-\alpha_{n}\right|,
\end{aligned}
$$

for some other $C(n, r)$ and $N(n, r)$. satisfy

In order to obtain (iii) of Proposition 2.2, the rational $\alpha_{n}=p / q$ have only to

that is,

$$
C(n, r) q^{N(n, r)}|\alpha-p / q|<2^{-n-r-1},
$$

\section{The terms}

$$
|\alpha-p / q|<2^{-n-r-1} C(n, r)^{-1} q^{-N(n, r)} .
$$

$$
\epsilon=2^{-n-r-1} C(n, r)^{-1} \text { and } N=N(n, r)
$$

are already determined beforehand or by the previous step of the induction. Since $\alpha$ is Liouville, there exists a rational $p / q$ which satisfies (2.1) for these values of $\epsilon$ and $N$. Setting it $p_{n} / q_{n}$, we establish (iii) for the $n$-th step of the induction.

In fact there are infinitely many choices of $p_{n} / q_{n}$. This enables us to assume more.

Remark 2.6. The denominator $q_{n}$ can be chosen as large as we want, compared with anything defined before the $n$-th step. 
To ensure the convergence of $H_{n}^{-1}$, notice that

$$
\left\|H_{n}^{-1}-H_{n-1}^{-1}\right\|_{0}=\left\|\left(h_{n}^{-1}-\mathrm{Id}\right) \circ H_{n-1}^{-1}\right\|_{0}=\left\|h_{n}^{-1}-\mathrm{Id}\right\|_{0} \leq q_{n}^{-1},
$$

where the last inequality follows from the fact that $h_{n}$ is a lift of some homeomorphism $\hat{h}_{n}$ by the $q_{n}$-fold cyclic covering map such that $\operatorname{Fix}\left(h_{n}\right) \neq \emptyset$, that is, $h_{n}$ is a periodic oscillation of period $q_{n}^{-1}$.

Therefore if we choose $q_{n}$ to grow rapidly (which is possible by Remark 2.6), then $H_{n}^{-1}$ converges to a continuous map $H^{-1}: S^{1} \rightarrow S^{1}$. But since $H^{-1}$, being a uniform limit of homeomorphisms, is monotone and $H^{-1} f=R_{\alpha} H^{-1}$, one can show that $H^{-1}$ is in fact a homeomorphism.

2.4. A variant of Method I. To show the $C^{\infty}$ density of $G_{1, \text { sing }}$, a slightly more complicated construction is convenient. Instead of setting $h_{n}$ to be a lift of $\hat{h}_{n}$ by the cyclic $q_{n}$-fold covering map, we can set $h_{n}$ to be the lift by the cyclic $Q_{n}$ covering, where $Q_{n}=K(n) q_{n}$, and $K(n)$ is a positive integer determined by the previous data, i. e. $\hat{h}_{1} \cdots \hat{h}_{n-1}$ and $q_{1}, \cdots, q_{n-1}$. Also in this case, (2.3) takes the form

$$
\left\|\left|h_{n}\right|\right\|_{n+r+1} \leq\left\|\left|\hat{h}_{n}\right|\right\|_{n+r+1}\left(K(n) q_{n}\right)^{n+r}=C(n, r) q_{n}^{N(n, r)} .
$$

So the estimates of (2.4) and (2.5) is of the same form although $C(n, r)$ is changed. Thus we can find a solution of (2.7).

2.5. Method II. In this method, we define $\alpha_{n}=p_{n} / q_{n}$ and $h_{n} \in F$ at the same time in the $n$-th step of the induction. First we consider a one parameter family $\left\{\hat{h}_{t}\right\}(t \in(0,1))$ in $F$ such that $\operatorname{Fix}\left(\hat{h}_{t}\right) \neq \emptyset$. We are mostly interested for small value of $t$. Assume for any $r \in \mathbb{N}$, there is a constant $C(r)>0$ and an integer $m(r)$ such that

$$
\left\|\left|\hat{h}_{t}\right|\right\|_{r} \leq C(r) t^{-m(r)}
$$

Let $\delta_{n}(n \in \mathbb{N})$ be a positive number. Define $h_{n}$ to be the lift of $\hat{h}_{q_{n}^{-\delta_{n}}}$, where $\alpha_{n}=p_{n} / q_{n}$ is going to be decided. Even in this case we have by (2.8)

$$
\begin{aligned}
\left\|\left|h_{n}\right|\right\|_{n+r+1} & \leq\left\|\left|\hat{h}_{q_{n}^{-\delta_{n}}}\right|\right\|_{n+r+1} q_{n}^{n+r} \\
& \leq C(r) q_{n}^{\delta_{n} m(r)+n+r},
\end{aligned}
$$

that is, the estimate of $\left\|\left|h_{n}\right|\right\|_{n+r+1}$ takes exactly the same form as in (2.3). Likewise that of $\left\|\left|h_{i}\right|\right\|_{n+r+1}(1 \leq i<n)$ is the same as in (2.2).

Therefore the inequality to decide $\alpha_{n}=p_{n} / q_{n}$ also takes the form of (2.7) and the Liouville property of $\alpha$ enables us to choose $\alpha_{n}$. Then the correspoding sequence $\left\{f_{n}\right\}$ converges to $f \in F_{\alpha}$ in the $C^{\infty}$-topology such that $d_{r}\left(f, R_{\alpha}\right)<2^{-r}$ for a given $r$, and also $H_{n}^{-1}$ uniformly to $H^{-1}$.

In the proof of the $C^{\infty}$-density of $G_{k}, \delta_{n}=1$ for any $n$. For $G_{\beta}, \delta_{n}$ is slightly varying. For $G_{0, \text { ac }}$, we start with a 2 parameter family, but this is explained later in the section for $G_{0, \text { ac }}$.

\section{The space $G_{1, \text { sing }}$}

The purpose of this section is to show that the space $G_{1 \text {,sing }}$ is $C^{\infty}$-dense in $F_{\alpha}$. We follow 2.4. Thus the sequence $\left\{\hat{h}_{n}\right\}$ is defined in the first place. Fix once 
and for all integers $k_{n} \in \mathbb{N}$ such that

$$
\prod_{i=1}^{\infty}\left(1-k_{i}^{-1}\right)>0 \text {. }
$$

For example, $k_{i}=(i+1)^{2}$. Let

$$
\hat{J}_{n}=\left[0,1-k_{n}^{-1}\right] \text { and } \hat{I}_{n}=\left[0, k_{n}^{-1}\right],
$$

and let $\hat{h}_{n} \in F$ be a diffeomorphism such that $\hat{h}_{n}\left(\hat{J}_{n}\right)=\hat{I}_{n}$ and $\hat{h}_{n}$ is linear on $\hat{J}_{n}$. Let

$$
Q_{n}=\left(k_{1} \cdots k_{n-1} q_{1} \cdots q_{n-1}\right) q_{n} .
$$

Consider the lift $h_{n}$ of $\hat{h}_{n}$ by the cyclic $Q_{n}$-fold covering map $\pi_{Q_{n}}$ such that $\operatorname{Fix}\left(h_{n}\right) \neq \emptyset$.

Following Paragraph 2.4, one can choose $\alpha_{n}=p_{n} / q_{n}$ inductively so that $f_{n}$ converges in the $C^{\infty}$ topology to $f \in F_{\alpha}$ such that $d_{r}\left(f, R_{\alpha}\right)<2^{-r}$ for a given $r \in \mathbb{N}$, and $H_{n}^{-1}=h_{n}^{-1} \cdots h_{1}^{-1}$ uniformly to a homeomorphism $H^{-1}$. Let

$$
J_{n}=\pi_{Q_{n}}^{-1}\left(\hat{J}_{n}\right) \text { and } I_{n}=\pi_{Q_{n}}^{-1}\left(\hat{I}_{n}\right) .
$$

Then we have $m\left(J_{n}\right)=1-k_{n}^{-1}$ and $m\left(I_{n}\right)=k_{n}^{-1}$, where $m$ denotes the Lebesgue measure. Given a finite subgoup $P$ of $S^{1}$, we call an interval in $S^{1}$ a $P$-interval if its endpoints are contained in $P$. Since $Q_{n}$ is a multiple of $k_{n-1} Q_{n-1}$, we have

(3.1) Any component of $J_{n-1}$ is a $\left(Q_{n}^{-1} \mathbb{Z}\right) / \mathbb{Z}$-interval.

Let

$$
C=\bigcap_{i=1}^{\infty} J_{i} \text { and } C_{n}=\bigcap_{i=1}^{n} J_{i}
$$

Then we have by $(3.1)$

$$
m\left(C_{n}\right)=\prod_{i=1}^{n}\left(1-k_{i}^{-1}\right) \text { and therefore } m(C)>0 .
$$

Now since $\left(Q_{n+1}^{-1} \mathbb{Z}\right) / \mathbb{Z}$ is pointwise fixed by $h_{j}(j>n)$, we also get by $(3.1)$

$$
h_{j}\left(J_{n}\right)=J_{n} \text { if } j>n .
$$

This implies that for any big $j$,

$$
H_{j}\left(J_{n}\right)=H_{n}\left(J_{n}\right)=H_{n-1} h_{n}\left(J_{n}\right)=H_{n-1}\left(I_{n}\right) .
$$

That is,

$$
J_{n}=H_{j}^{-1} H_{n-1}\left(I_{n}\right) .
$$

Therefore the uniform limit $H^{-1}$ of $H_{j}^{-1}$ satisfies for any $n \in \mathbb{N}$

$$
J_{n}=H^{-1}\left(H_{n-1}\left(I_{n}\right)\right) \text {, where } H_{0}=\mathrm{Id} .
$$

Thus we have

$$
H\left(C_{n}\right)=H\left(\bigcap_{i=1}^{n} J_{i}\right)=\bigcap_{i=1}^{n} H\left(J_{i}\right)=\bigcap_{i=1}^{n} H_{i-1}\left(I_{i}\right)
$$

Again (3.1) and the linearity of $\hat{h}_{n}$ on $\hat{J}_{n}$ enable us to compute the measure. We have

$$
m\left(H\left(C_{n}\right)\right)=\prod_{i=1}^{n} k_{i}^{-1} \rightarrow 0 \text { and therefore } m(H(C))=0 .
$$


Now the $f$-invariant measure $\mu_{f}=H_{*} m$ satisfies

$$
\mu_{f}(H(C))=\left(H_{*} m\right)(H(C))=m\left(H^{-1}(H(C))\right)=m(C)>0 .
$$

In summary we have

Clearly (3.2) implies that $\mu_{f}$ is not equivalent to $m$, that is, $H$ is singular.

In the rest we shall show that $H^{-1}$ is $d$-Hölder for any $d \in(0,1)$. For this purpose we do not need any further condition on the sequence $\left\{\hat{h}_{n}\right\}$. We just need to assume that the sequence $\left\{q_{n}\right\}$ grows fast compared with $\left\{\hat{h}_{n}\right\}$. Concretely let $M_{n}>1$ be a constant which satisfy

$$
M_{n}^{-1}|x-y| \leq\left|H_{n}^{-1}(x)-H_{n}^{-1}(y)\right| \leq M_{n}|x-y|, \quad \forall x, y \in S^{1} .
$$

We assume:

(3.2) For any $d \in(0,1)$, there exists $n_{0}$ such that if $n \geq n_{0}$ then $M_{n+1} Q_{n}^{d-1} \leq 1$.

(3.3) $Q_{n}^{-1} \leq 8^{-1} M_{n-1}^{-1} Q_{n-1}^{-1}$ for any $n \geq 0$.

(3.4) $Q_{n}^{-1} \leq 2^{-1} Q_{n-1}^{-1}$ for any $n \geq 0$.

Notice that the Lipschitz constant of $h_{j}$ is the same as that of $\hat{h}_{j}$, and therefore $M_{n+1}$ depends only on $\hat{h}_{1}, \cdots, \hat{h}_{n+1}$, which are determined in the very beginning of the argument. After that, we start the inductive step to choose the number $\alpha_{n}=p_{n} / q_{n}$. Thus (3.2) is attained if simply we choose $q_{n}$ big enough (and therefore $Q_{n}$ big) compared with the Lipschitz constants of $\hat{h}_{1}, \cdots, \hat{h}_{n+1}$, which is possible by Remark [2.6] Likewise (3.3) and (3.4) can be attained by choosing $q_{n}$ big compared with the previous data.

Now assume $n \geq n_{0}$ and $Q_{n+1}^{-1} \leq|x-y| \leq Q_{n}^{-1}$. Then

$$
\begin{aligned}
\left|H_{n+1}^{-1}(x)-H_{n+1}^{-1}(y)\right| & \leq M_{n+1}|x-y|=M_{n+1}|x-y|^{1-d}|x-y|^{d} \\
& \leq M_{n+1} Q_{n}^{d-1}|x-y|^{d} \leq|x-y|^{d} .
\end{aligned}
$$

On the other hand we have

$$
\left|H_{n+1}^{-1}(x)-H_{n+1}^{-1}(y)\right| \geq M_{n+1}^{-1}|x-y| \geq M_{n+1}^{-1} Q_{n+1}^{-1} .
$$

This means that the distance of the points $H_{n+1}^{-1}(x)$ and $H_{n+1}^{-1}(y)$ is big enough compared with the periods of periodic oscillations $h_{n+2}, h_{n+3}, \cdots$, and thus compared with the distance of the points $H_{n+1}^{-1}(x)$ and $H^{-1}(x)$.

To be precise, from (3.3) and (3.4) follows

$$
\sum_{i=n}^{\infty} Q_{i}^{-1} \leq 2 Q_{n}^{-1} \leq 4^{-1} M_{n-1}^{-1} Q_{n-1}^{-1} .
$$

Therefore

$$
\begin{aligned}
\left|H_{n+1}^{-1}(x)-H^{-1}(x)\right| & \leq \sum_{i=n+2}^{\infty} Q_{i}^{-1} \leq 4^{-1} M_{n+1}^{-1} Q_{n+1}^{-1} \\
\leq 4^{-1} M_{n+1}^{-1}|x-y| & \leq 4^{-1}\left|H_{n+1}^{-1}(x)-H_{n+1}^{-1}(y)\right|,
\end{aligned}
$$

and likewise for $y$. This implies

$$
\left|H^{-1}(x)-H^{-1}(y)\right| \leq 2\left|H_{n+1}^{-1}(x)-H_{n+1}^{-1}(y)\right| \leq 2|x-y|^{d} .
$$


We have shown the above for any $x$ and $y$ such that $d(x, y) \leq Q_{n_{0}}^{-1}$. Clearly this is enough for showing that $H^{-1}$ is $d$-Hölder.

The proof that $H$ is $d$-Hölder for any $d \in(0,1)$ uses the same condition (3.2) $\sim(3.4)$. The analogous argument is omitted.

\section{The space $G_{1, \text { ac }}$}

We start with a sequence $\left\{\hat{h}_{n}\right\}$ in $F$. This time $\hat{h}_{n}$ is a diffeomorphism supported on $\left[0,2^{-n-1}\right]$ such that $\left\|\hat{h}_{n}^{\prime}\right\|_{0}>n$. We define $h_{n}$ to be the lift of $\hat{h}_{n}$ by the $q_{n}$-fold cyclic covering map $\pi_{q_{n}}$ such that Fix $\left(h_{n}\right) \neq \emptyset$. As is exposed in 2.3. Method I, we can choose $\alpha_{n}=p_{n} / q_{n}$ inductively such that $f_{n}$ converges in the $C^{\infty}$ topology to $f \in F_{\alpha}$ with $d_{r}\left(f, R_{\alpha}\right)<2^{-r}$ for a given $r \in \mathbb{N}$, and $H_{n}^{-1}=h_{n}^{-1} \cdots h_{1}^{-1}$ uniformly to a homeomorphism $H^{-1}$.

Just as in the last part of the previous section, $H^{ \pm 1}$ is $d$-Hölder for any $d \in(0,1)$ if we choose $\left\{q_{n}\right\}$ to grow fast compared with $\left\{\hat{h}_{n}\right\}$.

Next let us show that $H$ is absolutely continuous. Let

$$
\hat{K}_{n}=\left[2^{-n-1}, 1\right] \text { and } K_{n}=\pi_{q_{n}}^{-1}\left(\hat{K}_{n}\right) .
$$

Then $h_{n}$ is the identity on $K_{n}$. Let $X=\cap_{n=1}^{\infty} K_{n}$. Then we have

$$
m(X) \geq 1-\sum_{n=1}^{\infty} m\left(S^{1} \backslash K_{n}\right) \geq 1-\sum_{n=1}^{\infty} 2^{-n-1} \geq 2^{-1} .
$$

Clearly $H$ is the identity on the positive measure set $X$. This implies that for any Bore subset $B, \mu_{f}(B \cap X)=m(B \cap X)$ and that $\mu_{f}(X)=m(X)>0$.

Then the invariant measure $\mu_{f}$ must be equivalent to $m$. For, if not, $\mu_{f}$ is singular to $m$, i. e. there is a Borel subset $B$ such that $m(B)=1$ and $\mu_{f}(B)=0$. But then $m(B \cap X)=m(X)>0$ and $\mu_{f}(B \cap X)=0$. A contradiction shows that $H$ is absolutely continuous.

Finally let us show that $H$ is not $C^{1}$, which implies that $H$ is not bi-Lipschitz by Remark 1.2 Let $\hat{I}_{n}$ be a closed interval such that $\hat{h}_{n}^{\prime}>n$ on $\hat{I}_{n}$, and $I_{n}=\pi_{q_{n}}^{-1}\left(\hat{I}_{n}\right)$. Thus $h_{n}^{\prime}>n$ on $I_{n}$. Now if $q_{n}$ grows sufficiently fast, then for each $i \in \mathbb{N}$ and for any component $K_{i}^{0}$ of $K_{i}$, there is a component $K_{i+1}^{0}$ of $K_{i+1}$ such that $K_{i+1}^{0} \subset K_{i}^{0}$.

This shows that there is a component $K_{n-1}^{0}$ of $K_{n-1}$ which is contained in $\bigcap_{i=1}^{n-1} K_{i}$. Clearly $H_{n-1}=\operatorname{Id}$ on $K_{n-1}^{0}$. Now if $q_{n}$ is big enough, there is a component $I_{n}^{0}$ of $I_{n}$ which is contained in $K_{n-1}^{0}$. On $I_{n}^{0}$, we have $H_{n}=h_{n}$.

Moreover if $q_{n+1}, q_{n+2}, \cdots$ are big enough compared with the length of $I_{n}^{0}$, and grow fast, then if we put

$$
Z_{n}=I_{n}^{0} \cap \bigcap_{i=n+1}^{\infty} K_{i},
$$

we have $m\left(Z_{n}\right)>0$. Notice that we can assume this for any $n$. Clearly $H=h_{n}$ on $Z_{n}$. Now consider the set $Z_{n}^{*}$ of the points of density of the positive measure set $Z_{n}$. The set $Z_{n}^{*}$ is perfect in the sense that $Z_{n}^{*}$ is contained in the derived set of $Z_{n}^{*}$. Since $Z_{n}^{*}$ is contained in the closure of $Z_{n}, H=h_{n}$ on $Z_{n}^{*}$. Moreover since $Z_{n}^{*}$ is perfect, $H^{\prime}=h_{n}^{\prime}$ if $H$ is $C^{1}$. That is, $H^{\prime}>n$ on $Z_{n}^{*}$ if we assume 
$H$ is a $C^{1}$ diffeomorphism. Since $n$ is arbitrary, this implies that $H$ is not a $C^{1}$ diffeomorphsim.

\section{The space $G_{\beta}$}

Here we follow 2.5 Method II. Consider a $C^{\infty}$ bump function $\psi: \mathbb{R} \rightarrow[0,1]$ such that $\psi\left(\left(-\infty,-4^{-1}\right]\right)=\{0\}, \psi\left(\left[4^{-1}, \infty\right)\right)=\{1\}$, and $\psi$ is strictly monotone increasing on $\left[-4^{-1}, 4^{-1}\right]$. For any $t \in(0,1)$, define a one parameter family $\left\{\hat{h}_{t}\right\}$ in $F$ obtained by smoothly joining the two affine functions, one $x \mapsto t^{-1} x$ on $[0, t]$ and the other $x \mapsto t(x-1)+1$ on $[0,1]$. Notice that the two functions coincide at $x=t(1+t)^{-1}$, and the latter is transfered to $x \mapsto t x$ on $[-1,0]$. Precisely we define $\hat{h}_{t}$ as follows.

(i) If $x \in\left[-4^{-1} t, 4^{-1} t\right], \quad \hat{h}_{t}(x)=\left(1-\psi\left(t^{-1} x\right)\right) t x+\psi\left(t^{-1} x\right) t^{-1} x$.

(ii) If $x \in\left[4^{-1} t, t(1+t)^{-1}-4^{-1} t\right], \quad \hat{h}_{t}(x)=t^{-1} x$.

(iii) If $x \in\left[t(1+t)^{-1}-4^{-1} t, t(1+t)^{-1}+4^{-1} t\right]$,

$\hat{h}_{t}(x)=\left(1-\psi\left(t^{-1} x-(t+1)^{-1}\right)\right) t^{-1} x+\psi\left(t^{-1} x-(t+1)^{-1}\right)(t(x-1)+1)$.

(iv) If $x \in\left[t(1+t)^{-1}+4^{-1} t, 1-4^{-1} t\right], \quad \hat{h}_{t}(x)=t(x-1)+1$.

Since we have used affine conjugation of the same bump function, a routine computation shows that $\hat{h}_{t}$ satisfies (2.8) of Paragraph 2.5.

Fix once and for all a sequence $\beta_{n} \downarrow \beta$, and set

$$
t_{n}=q_{n}^{1-\beta_{n}^{-1}} \text {. }
$$

Define $h_{n}$ to be the lift of $\hat{h}_{t_{n}}$ by the cyclic $q_{n}$-covering, where $\alpha_{n}=p_{n} / q_{n}$ is to be determined by (2.7) in Sect. 2 .

Since we can choose $q_{n}$ big enough compared with the previous data, we can assume the following.

$$
t_{1}^{-1} \cdots t_{n-1}^{-1} q_{n}^{-1+\beta \beta_{n}^{-1}} \leq 1 .
$$

Notice that (5.1) implies that

$$
q_{n}^{-1}=\left(t_{n} q_{n}^{-1}\right)^{\beta_{n}}, \text { and especially } q_{n}^{-1}<\left(t_{n} q_{n}^{-1}\right)^{\beta} \text {. }
$$

Define

$$
\hat{I}_{t}=[t / 4,7 t / 12],
$$

and $I_{n}=\pi_{q_{n}}^{-1}\left(\hat{I}_{t_{n}}\right)$. Notice that each component of $I_{n}$ has length $3^{-1} t_{n} q_{n}^{-1}$, and $h_{n}$ is an affine transformation with magnification $t_{n}^{-1}$ on $I_{n}$ for any small $t_{n}$.

Let us show first of all that $H$ is not $d$-Hölder for any $d \in(\beta, 1)$. If one chooses $q_{n}$ to grow fast, then for any component $I_{i}^{0}$ of $I_{i}$, there is a component $I_{i+1}^{0}$ of $I_{i+1}$ such that $I_{i+1}^{0} \subset I_{i}^{0}$. This shows that there is a component $I_{n}^{0}$ of $I_{n}$ which is contained in $\bigcap_{i=1}^{n} I_{i}$. On $I_{n}^{0}, H_{n}$ is an affine transformation of magnification $t_{1}^{-1} \cdots t_{n}^{-1}$. Let $I_{n}^{0}=\left[x^{\prime}, y^{\prime}\right]$.

Denote $H^{(n+1)}=H_{n}^{-1} H$. Its inverse $\left(H^{(n+1)}\right)^{-1}$ is the uniform limit of

$$
h_{n+m}^{-1} \cdots h_{n+2}^{-1} h_{n+1}^{-1}
$$

as $m \rightarrow \infty$, and $h_{n+1}, h_{n+2}, \cdots$ are periodic oscillations of small periods $q_{n+1}^{-1}, q_{n+2}^{-1}, \cdots$. 
Define $x, y \in S^{1}$ by

$$
H^{(n+1)}(x)=x^{\prime} \text { and } H^{(n+1)}(y)=y^{\prime} .
$$

It is possible to choose $\left\{q_{n}\right\}$ so as to satisfy

$$
\sum_{i=n+1}^{\infty} q_{i}^{-1} \leq 3^{-1} t_{n} q_{n}^{-1}
$$

for any $n \in \mathbb{N}$. (Decompose this into two inequalities as in the previous section, and ressort to Remark 2.6.) Then we have

$$
|x-y| \leq 3\left|x^{\prime}-y^{\prime}\right| .
$$

Now it follows that

$$
\begin{gathered}
|H(x)-H(y)|=\left|H_{n}\left(x^{\prime}\right)-H_{n}\left(y^{\prime}\right)\right|=t_{1}^{-1} \cdots t_{n}^{-1}\left|x^{\prime}-y^{\prime}\right| \\
=t_{1}^{-1} \cdots t_{n}^{-1} \cdot 3^{-1} t_{n} q_{n}^{-1}=3^{-1} t_{1}^{-1} \cdots t_{n-1}^{-1} q_{n}^{-1}
\end{gathered}
$$

and that

$$
|x-y|^{d} \leq 3^{d}\left|x^{\prime}-y^{\prime}\right|^{d}=3^{d}\left(3^{-1} t_{n} q_{n}^{-1}\right)^{d}=t_{n}^{d} q_{n}^{-d} .
$$

Now a computation using (5.1) shows that if $\beta_{n}<d$,

$$
\begin{aligned}
|H(x)-H(y)| /|x-y|^{d} & \geq 3^{-1} t_{1}^{-1} \cdots t_{n-1}^{-1} q_{n}^{d-1} t_{n}^{-d} \\
& =3^{-1} t_{1}^{-1} \cdots t_{n-1}^{-1} q_{n}^{d-1}\left(q_{n}^{1-\beta_{n}^{-1}}\right)^{-d} \\
& =3^{-1} t_{1}^{-1} \cdots t_{n-1}^{-1} q_{n}^{\beta_{n}^{-1} d-1} \\
& \geq 3^{-1} t_{1}^{-1} \cdots t_{n-1}^{-1} .
\end{aligned}
$$

Since the RHS of this expression can be arbitrarily large, we have shown that $H$ is not $d$-Hölder for any $d \in(\beta, 1)$.

We next show that $H^{-1}$ is $\beta$-Hölder. Assume for some $n \in \mathbb{N}$,

$$
t_{n+1} q_{n+1}^{-1} \leq|x-y| \leq t_{n} q_{n}^{-1} .
$$

Then we have

$$
\left|H_{n}^{-1}(x)-H_{n}^{-1}(y)\right| \leq|x-y|^{\beta} .
$$

In fact, since the Lipschitz constant of $h_{i}^{ \pm}$is $t_{i}^{-1}$

$$
\begin{aligned}
\left|H_{n}^{-1}(x)-H_{n}^{-1}(y)\right| & \leq t_{1}^{-1} \cdots t_{n}^{-1}|x-y| \\
& =t_{1}^{-1} \cdots t_{n}^{-1}|x-y|^{1-\beta}|x-y|^{\beta} \\
& \leq t_{1}^{-1} \cdots t_{n}^{-1}\left(t_{n} q_{n}^{-1}\right)^{1-\beta}|x-y|^{\beta} \\
& =t_{1}^{-1} \cdots t_{n-1}^{-1} t_{n}^{-\beta} q_{n}^{\beta-1}|x-y|^{\beta} \\
& =t_{1}^{-1} \cdots t_{n-1}^{-1}\left(q_{n}^{1-\beta_{n}^{-1}}\right)^{-\beta} q_{n}^{\beta-1}|x-y|^{\beta} \\
& =t_{1}^{-1} \cdots t_{n-1}^{-1} q_{n}^{-1+\beta \beta_{n}^{-1}}|x-y|^{\beta} .
\end{aligned}
$$

Thus (5.5) follows from (5.2).

Now the rest of the proof is divided into two cases.

CASE $1 \quad\left|H_{n}^{-1}(x)-H_{n}^{-1}(y)\right| \geq q_{n+1}^{-1}$. 
Since $h_{n+1}^{-1}$ is a periodic osillation of period $q_{n+1}^{-1}$, it follows from the assumption of Case 1 that

$$
\left|H_{n+1}^{-1}(x)-H_{n+1}^{-1}(y)\right| \leq 2\left|H_{n}^{-1}(x)-H_{n}^{-1}(y)\right| .
$$

If we choose $q_{n+2}, q_{n+3}, \cdots$ to grow fast, then we have

$$
\left|H^{-1}(x)-H^{-1}(y)\right| \leq 3\left|H_{n}^{-1}(x)-H_{n}^{-1}(y)\right| \leq 3|x-y|^{\beta},
$$

finishing the proof in this case.

CASE $2 \quad\left|H_{n}^{-1}(x)-H_{n}^{-1}(y)\right| \leq q_{n+1}^{-1}$.

Since $h_{n+1}^{-1}$ is a periodic oscillation of period $q_{n+1}^{-1}$, we have in this case that

$$
\left|H_{n+1}^{-1}(x)-H_{n+1}^{-1}(y)\right| \leq q_{n+1}^{-1}
$$

and therefore we get using (5.3)

$$
\left|H^{-1}(x)-H^{-1}(y)\right| \leq 2 q_{n+1}^{-1} \leq 2\left(t_{n+1} q_{n+1}^{-1}\right)^{\beta} \leq 2|x-y|^{\beta},
$$

and the proof is complete.

Finally let us show that $H$ is $\beta$-Hölder. Again we assume for some $n \in \mathbb{N}$,

$$
t_{n+1} q_{n+1}^{-1} \leq|x-y| \leq t_{n} q_{n}^{-1} .
$$

Let

$$
x^{\prime}=H^{(n+2)}(x), \text { and } y^{\prime}=H^{(n+2)}(y) .
$$

If $q_{n+2}^{-1}, q_{n+3}^{-1}, \cdots$ are small enough, then we have

$$
\left|x^{\prime}-y^{\prime}\right| \leq 2|x-y|
$$

CASE $1\left|x^{\prime}-y^{\prime}\right| \geq q_{n+1}^{-1}$.

If we put

$$
x^{\prime \prime}=h_{n+1} x^{\prime} \text { and } y^{\prime \prime}=h_{n+1} x^{\prime},
$$

then by the assumption of Case 1 , we have

$$
\left|x^{\prime \prime}-y^{\prime \prime}\right| \leq 2\left|x^{\prime}-y^{\prime}\right| \text {. }
$$

We can show by the same computation as for $H^{-1}$ which stems from the condition $|x-y| \leq t_{n} q_{n}^{-1}$, that

$$
\left|H_{n}\left(x^{\prime \prime}\right)-H_{n}\left(y^{\prime \prime}\right)\right| \leq 4 t_{1}^{-1} \cdots t_{n}^{-1}|x-y| \leq 4|x-y|^{\beta},
$$

completing the proof in this case.

CASE $2\left|x^{\prime}-y^{\prime}\right| \leq q_{n+1}^{-1}$.

As before we have $\left|x^{\prime \prime}-y^{\prime \prime}\right| \leq q_{n+1}^{-1}$, where $x^{\prime \prime}$ and $y^{\prime \prime}$ are defined by (5.6). Now we have

$$
\begin{gathered}
|H(x)-H(y)|=\left|H_{n}\left(x^{\prime \prime}\right)-H_{n}\left(y^{\prime \prime}\right)\right| \\
\leq t_{1}^{-1} \cdots t_{n}^{-1}\left(q_{n+1}^{-1}\right)^{1-\beta \beta_{n+1}^{-1}}\left|x^{\prime \prime}-y^{\prime \prime}\right|^{\beta \beta_{n+1}^{-1}} .
\end{gathered}
$$

Therefore by (5.2), we have

$$
|H(x)-H(y)| \leq\left|x^{\prime \prime}-y^{\prime \prime}\right|^{\beta \beta_{n+1}^{-1}}
$$

On the other hand by (5.3),

$$
\left|x^{\prime \prime}-y^{\prime \prime}\right| \leq q_{n+1}^{-1}=\left(t_{n+1} q_{n+1}^{-1}\right)^{\beta_{n+1}} \leq|x-y|^{\beta_{n+1}} .
$$

The proof that $G_{\beta}$ is $C^{\infty}$-dense is now complete. 


\section{The space $G_{0, \text { ac }}$}

Given $0<s<t<1$, consider the three affine transformations:

- $x \mapsto s^{-1} t x$ for $x \in[0, s]$.

- $x \mapsto s t^{-1}(x-t)+t$ for $x \in[0, t]$.

- $x \mapsto x$ for $x \in[t, 1]$.

Define a family $\hat{h}_{s, t}$ in $F$ which smoothly joins the above three transformations by bump functions. On the interval $\hat{I}_{s, t}=[s / 4,7 s / 12], \hat{h}_{s, t}$ is to be an affine transformation of magnification $s^{-1} t$. We also have $\hat{h}_{s, t}=$ Id on the interval $\hat{K}_{s, t}=$ $[2 t, 1]$. If we choose the bump functions to be affine conjugates of the same function, as we did in Sect. 5, we have

$$
\left\|\left|\hat{h}_{s, t}\right|\right\|_{r} \leq C(r) s^{-m(r)} t^{-m(r)}
$$

for some $C(r)$ and $m(r)$.

Let

$$
t=3^{-n-2}
$$

and $q_{n} \in \mathbb{N}$ be arbitrary. Let $h_{n}$ be the lift of $\hat{h}_{s, t}$ by the $q_{n}$-fold cyclic covering $\pi_{q_{n}}$ such that $\operatorname{Fix}\left(h_{n}\right) \neq \emptyset$, and let $[x, y]$ be a component of $I_{n}=\pi_{q_{n}}^{-1}\left(\hat{I}_{s, t}\right)$. Also let $K_{n}=\pi_{q_{n}}^{-1}\left(\hat{K}_{s, t}\right)$.

Then for any $d \in(0,1)$, we have

$$
\frac{h_{n}(x)-h_{n}(y)}{(x-y)^{d}}=\frac{3^{-n-3} q_{n}^{-1}}{\left(3^{-1} s q_{n}^{-1}\right)^{d}}
$$

Setting the above ratio to be equal to $n$ and $d=n^{-1}$, we get

$$
s=3^{-n^{2}-3 n+1} n^{-n} q_{n}^{-n+1} .
$$

If we fix the value of $t$ and $s$ by (6.2) and (6.4), then (6.1) implies that for any $r \in \mathbb{N}$

$$
\left\|\left|h_{n}\right|\right\|_{n+r+1} \leq C(n, r) q_{n}^{N(n, r)},
$$

for some constants $C(n, r)$ and $N(n, r)$. Therefore we can follow 2.5. Method II, and can choose $\alpha_{n}=p_{n} / q_{n}$ inductively so that $f_{n}$ converges in the $C^{\infty}$ topology to $f \in F_{\alpha}$ in such a way that $d_{r}\left(f, R_{\alpha}\right)<2^{-r}$ for a given $r \in \mathbb{N}$, and $H_{n}^{-1}=h_{n}^{-1} \cdots h_{1}^{-1}$ uniformly to a homeomorphism $H^{-1}$.

Just as in Sect. 4 , if we choose $q_{n}$ to grow fast, then there is a component $I_{n}^{0}$ of $I_{n}$ such that $m\left(Z_{n}\right)>0$, where

$$
Z_{n}=I_{n}^{0} \cap \bigcap_{i \neq n} K_{i}
$$

For any distinct points $x, y \in Z_{n}$, we have

$$
\frac{H(x)-H(y)}{(x-y)^{n^{-1}}}=\frac{h_{n}(x)-h_{n}(y)}{(x-y)^{n^{-1}}}=n,
$$

since we have set the value of (6.3) to be equal to $n$. This shows that $H$ is not $d$-Hölder for any $d \in(0,1)$.

Finally the same argument as in Sect. 4 shows that $H$ is absolutely continuous. 


\section{The space $G_{k}$}

In this section we shall show that $G_{k}$ in Definition 1.4 is $C^{\infty}$-dense in $F_{\alpha}$. We consider the one parameter family $\left\{\hat{h}_{t}\right\}_{0<t<1}$ given by Lemma 7.1 below, and follows 2.5. Method II for the choice of $\delta_{n}=1(\forall n \in \mathbb{N})$, that is, we define $h_{n}$ to be the lift of $\hat{h}_{q_{n}^{-1}}$ by the cyclic $q_{n}$-fold covering such that $\operatorname{Fix}\left(h_{n}\right) \neq \emptyset$.

Lemma 7.1. Given $k \in \mathbb{N}$, there exists a one parameter family $\left\{\hat{h}_{t}\right\}_{0<t<1}$ in $F$ which satisfies the following properties.

(i) $\hat{h}_{t}=$ Id in an interval $\hat{K}_{t}$ of length $\geq 1-t^{k}$.

(ii) $\left\|\hat{h}_{t}^{ \pm}-\mathrm{Id}\right\|_{k} \leq C t^{k}$ for some constant $C>0$.

(iii) $\left|\left(\hat{h}_{t}^{-1}\right)^{(k+1)}\right| \geq C^{-1}$ on an interval $\hat{I}_{t}$ for some constant $C>0$.

(iv) For any $r \in \mathbb{N},\left\|\left|\hat{h}_{t}\right|\right\|_{r} \leq C_{r} t^{-m(k, r)}$ for some constant $C_{r}>0$ and an integer $m(k, r)$.

The proof of this lemma is postponed until the end of this section.

By following 2.5. Method II, thanks to (iv) above, we can choose $\alpha_{n}=p_{n} / q_{n}$ inductively so that $f_{n}$ converges in the $C^{\infty}$ topology to $f \in F_{\alpha}$ in such a way that $d_{r}\left(f, R_{\alpha}\right)<2^{-r}$ for a given $r \in \mathbb{N}$, and $H_{n}^{-1}=h_{n}^{-1} \cdots h_{1}^{-1}$ uniformly to a homeomorphism $H^{-1}$.

Let us show that a bit more careful choice of $\alpha_{n}$ ensures that $H^{-1}$ is a $C^{k}$ diffeomorphism. Now (ii) above and Lemma 2.5 implies that

$$
\left\|h_{n}^{-1}-\mathrm{Id}\right\|_{k}=\left\|\hat{h}_{q_{n}^{-1}}^{-1}-\mathrm{Id}\right\|_{k} \cdot q_{n}^{k-1} \leq C q_{n}^{-1} .
$$

By Lemma 2.3 we have

$$
d_{k}\left(H_{n}^{-1}, H_{n-1}^{-1}\right) \leq C\left\|h_{n}^{-1}-\mathrm{Id}\right\|_{k}\left\|\left|H_{n-1}\right|\right\|_{k}^{k} \leq C q_{n}^{-1}\left\|\left|H_{n-1}\right|\right\|_{k}^{k} .
$$

Thus if we choose $q_{n}$ big enough compared with $\left\|\left|H_{n-1}\right|\right\|_{k}^{k}$, then we have

$$
d_{k}\left(H_{n}^{-1}, H_{n-1}^{-1}\right) \leq 2^{-n} .
$$

This shows that the limit $H^{-1}$ is a $C^{k}$ diffeomorphism.

Let us show that $H^{-1}$ is not a $C^{k+1}$ diffeomorphism. Let

$$
K_{n}=\pi_{q_{n}}^{-1}\left(\hat{K}_{q_{n}^{-1}}\right) \text { and } I_{n}=\pi_{q_{n}}^{-1}\left(\hat{I}_{q_{n}^{-1}}\right) .
$$

Thus $h_{n}=\operatorname{Id}$ on $K_{n}$ and $\left|\left(h_{n}^{-1}\right)^{(k+1)}\right|>C^{-1} q_{n}^{k}$ on $I_{n}$, the latter by (iii) above. The rest of the proof is nearly the same as in Sect. 4, where we have shown that $H$ is not $C^{1}$.

Now let us prove Lemma 7.1. We begin with the following lemma found in $[\mathbf{H}$, p. 154. The proof is included for the convenience of the reader.

Lemma 7.2. Let $\left\{\phi_{a}\right\}$ be a $C^{\infty}$ flow on $S^{1}$. For any $r \geq 1$, there is a constant $C_{r}>0$ such that for any $a \in[-1,1]$, we have

$$
C_{r}^{-1}|a| \leq\left\|\left(\phi_{a}\right)^{(r)}\right\|_{0} \leq C_{r}|a| \text {. }
$$

Proof. Let

$$
C_{r}^{\prime}=\sup _{a \in[0,1]}\left\|\frac{\partial}{\partial a} \phi_{a}^{(r)}\right\|_{0}
$$


Then since $\phi_{0}^{(r)}=0$, we have by the mean value theorem

$$
|a|^{-1}\left\|\phi_{a}^{(r)}\right\|_{0} \leq C_{r}^{\prime}
$$

To show the converse inequality, consider $X=\left.\frac{\partial}{\partial a} \phi_{a}\right|_{a=0}$, the infinitesimal generator of the flow $\left\{\phi_{a}\right\}$. Then $X^{(r)}=\left.\frac{\partial}{\partial a} \phi_{a}^{(r)}\right|_{a=0}$ is not constantly equal to zero. Assume for some $x \in S^{1}$

$$
\left|\frac{\partial}{\partial a} \phi_{a}^{(r)}(x)\right|_{a=0} \mid=2 C_{r}^{\prime \prime}>0
$$

Then, by the definition of $\partial / \partial a$, there is $\alpha>0$ such that if $|a| \leq \alpha$,

$$
|a|^{-1}\left|\phi_{a}^{(r)}(x)\right| \geq C_{r}^{\prime \prime} .
$$

That is,

$$
|a|^{-1}\left\|\phi_{a}^{(r)}\right\|_{0} \geq C_{r}^{\prime \prime}
$$

For $\alpha \leq|a| \leq 1,|a|^{-1}\left\|\phi_{a}^{(r)}\right\|_{0}$ is positive and continuous in $a$. The proof is complete by relaxing the constant $C_{r}^{\prime}$ and $\left(C_{r}^{\prime \prime}\right)^{-1}$.

Choose a flow $\left\{\phi_{a}\right\}$ whose support is contained in $(0,1) \subset S^{1}$. For any $k \in \mathbb{N}$ and $t \in(0,1)$ let

$$
\hat{h}_{t}(x)=t^{k} \phi_{t^{k^{2}}}\left(t^{-k} x\right) .
$$

Then it is routine to check the properties in Lemma 7.1

\section{References}

[D] A. Denjoy, Sur les courbes défini par les équations différentielle à la surfase du tore. J. Math. Pures Appl. 9(11) (1932), 333-375.

[FS] B. Fayad and M. Saprykina, Weak mixing disc and annulus diffeomorphisms with arbitrary Liouvillean rotation number on the boundary, Ann. Sci. Ecole Norn. Sup. 38(2005), no. 3, 339-364.

[H] M. R. Herman, Sur la conjugaison diff'erentiable des difféomorphismes du cercle a des rotations, Publ. I. H. E. S. 49(1979), 5-233.

$[\mathrm{KH}]$ A. Katok and B. Hasselblatt, Introduction to the modern theory of dynamical systems, Encyclopedia of Mathematics and its Applications, Vol. 54, Cambridge University Press 1995.

[M] S. Matsumoto, A generic dimensional properties of the invariant measures for circle diffeomorphisms, Preprints. arXiv:1101.4463.

[P] Ya. Pesin, Dimension theory in dynamical systems: Contemporary views and applications. The university of Chicago Press, Chicago and London.

[S] V. Sadovskaya, Dimensional characteristics of invariant measures for circle diffeomorphisms, Erg. Th. Dyn. Sys. 29(2009), no.6, 1979-1992.

[Y1] J.-C. Yoccoz, Conjugaison différentiable des difféimorphismes du cercle dont le nombre de rotation vérifie une conditon diophantinne, Ann. Sci. Ecole Norm. Sup. 17(1984), no. 3, 333-359.

[Y2] J.-C. Yoccoz, Centralisateurs et conjugaison différentiable des difféomorphismes du cercle, Astérisque 231(1995), 89-242.

[Y3] L. S. Young, Dimension, entropy, and Lyapunov exponents, Erg. Th. Dyn. Sys. 2(1982), 109-124.

Department of Mathematics, College of Science and Technology, Nihon UniverSity, 1-8-14 Kanda, Surugadai, ChiYoda-ku, Tokyo, 101-8308 Japan

E-mail address: matsumo@math.cst.nihon-u.ac.jp 